\title{
Nautical Charts Generalization: A Pragmatic Approach
}

\author{
Andriani Skopeliti ${ }^{\text {a, }}$, , Lysandros Tsoulos ${ }^{\text {a }}$, Leda Stamou ${ }^{\text {a }}$, Shachak Pe'eri ${ }^{b}$ \\ ${ }^{a}$ Cartography Laboratory, School of Rural and Surveying Engineering, National Technical University of Athens, 15780 Zografou, \\ Greece,askop@survey.ntua.gr,lysandro@central.ntua.gr,lestamou@central.ntua.gr \\ ${ }^{b}$ National Ocean Service NOAA Office of Coast Survey; Silver Spring, MD 20910, USA, shachak.peeri@NOAA.gov \\ * Corresponding author
}

Keywords: Soundings; Depth Contours; Coastline; Generalization; Nautical chart; Electronic Nautical Chart - ENC; IHO standards; Digital Elevation Model - DEM

\begin{abstract}
:
Recent advances in Big Data technologies and the abundance of marine geospatial data collected and stored by Hydrographic Offices (HOs) require efficient generalization methods to support nautical chart production for a number of scales. Portrayed depths and depth contours are derived from soundings acquired by tidally-referenced surveys, also known as hydrographic surveys, which survey the seafloor and detect objects that are hazardous to navigation (e.g., rocks and wrecks). Common survey technologies used to map the seafloor include acoustic technologies (e.g., Multibeam Echo Sounders - MBES) and optical technologies (e.g., Airborne Lidar Bathymetry - ALB). In addition to bathymetry, highresolution, accurate coastal data are obtained from detailed Lidar surveys, high-resolution tidally-referenced imagery, and topographic surveys. Although one would expect a fully automated cartographic generalization process to be already available, it is still a (semi-)manual process. This is due to the volume of data, the complexity of the seafloor and the number of rules and constraints that ensure safe navigation. This paper proposes methods for soundings, depth contours and coastline generalization for Harbour and Approach Nautical Charts in a GIS environment in accordance with the International Hydrographic Organization (IHO) standards and widely adopted cartographic practices.
\end{abstract}

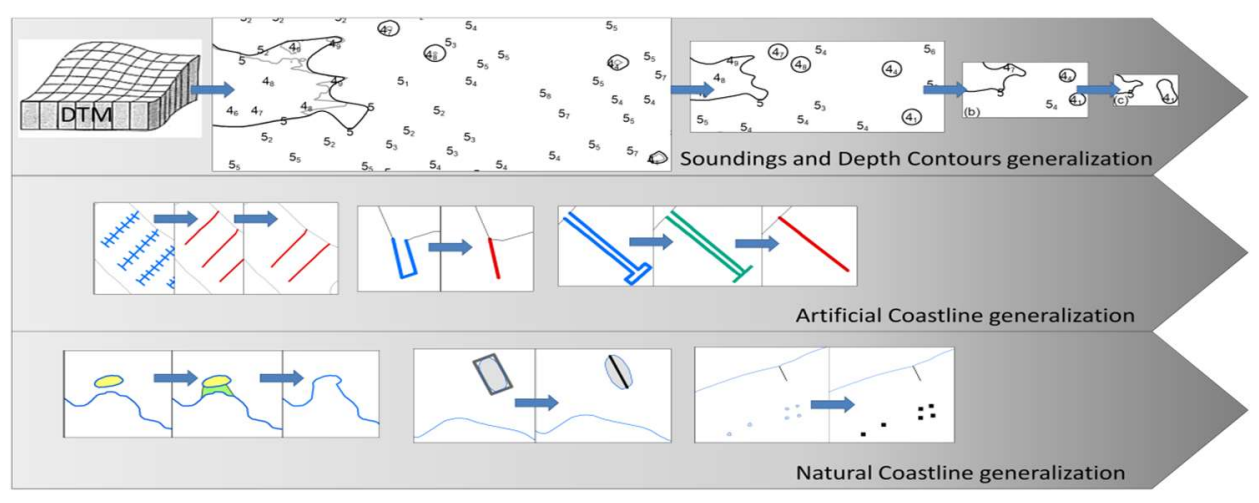

Figure 1. This paper proposes methods for soundings, depth contours and coastline generalization.

The proposed method for soundings generalization (Skopeliti et al., 2020) can be successfully applied to regularly spaced depth points extracted from a high-resolution DEM, which is usually the result of contemporary bathymetry collection methods. It can also be applied to irregularly spaced depths extracted from a larger scale nautical chart. It is based on three pillars: a. Soundings Pattern: The sounding selection method aims to a rhomboidal pattern that is used as a reference for locating soundings that are candidates for selection. The rhombus cell size can be adjusted for density and location of the soundings according to the chart scale, b. Classification of soundings: The selection of soundings for depiction at smaller scales is based on their classification into Prime, Background, Fill and Morphology soundings, c. Seabed Structure and Depth Integrity: The description of seabed surface is based on depth contours extracted from the DEM and generalized at the chart compilation scale. Scale-specific depth contours are generated by generalizing the raw depth contours extracted from the DTM. Consequently, the seabed description is scale-dependent and contributes to a scale-specific selection of soundings. This makes it superior to other seabed description methods, e.g., slope, feature recognition etc., which focus on details that are not relevant to the map compilation scale.

For coastline generalization (Skopeliti et al., 2021), the natural and artificial coastlines are processed separately. Although generalization of natural coastline is an open research topic, in this study, generalization focuses on its resolution and possible amalgamation or collapse of islands while maintaining their inherent characteristics. Appropriate operators for 
islands are invoked based on their area, dimensions and distance from the mainland coastline. In the context of generalization of artificial coastlines, a novel process is introduced by identifying the shape and form of the artificial structures as perceived by humans (e.g., a pier that looks like a T-shape) through pre-processing. First, all connected segments that form an artificial structure are evaluated. Then, each artificial structure is classified based on its specific characteristics regarding shape. For example, a T-shape pier is classified based on its inherent characteristics, e.g., length, width, and other characteristics identified through pre-processing, e.g., distance from the shoreline, azimuth with respect to a specific reference, etc. For each structure, elements that would change through generalization are identified, e.g., a T-shaped structure is analyzed to its vertical axis and cup. These elements are not originally semantically labelled, since structures are stored as simple line segments. Finally, generalization is performed according to the specifications.

For generalization of depth contours, it is important to identify the structure of the seabed to obtain the morphology. Unlike existing methods, in this study, each contour is first pre-processed and enriched with auxiliary information that helps to describe and generalize the seafloor (Skopeliti et al., 2021). For each contour, a set of properties is assessed that include: Geometry (e.g., open/closed), relative position to an open contour (e.g., shallow area/deep area), depths within (e.g., peaks/pits), and topological relationships between closed depth contours (e.g., not contained/not within, contain others, within others). Taking advantage of this structural description and proximity between depth contours, aggregation and exaggeration is performed. Depending on the line granularity, additional simplification and/or smoothing can be applied to the resulting depth contours as required. Each depth contour line should be generalized individually. Generalization begins with processing the shallow depth contours and proceeds with the deep ones. This is in accordance with the general principle of ensuring safe navigation to ensure the seaward displacement of the generalized depth contours into the deep areas. Therefore, each contour depends on the already generalized shallower depth contours. One of the most important properties of a nautical chart is the logical consistency between the features shown on the chart. Depth contours and soundings are derived from the same data source. However, an incompatibility can be observed in cases where a contour does not contain all soundings with depths corresponding to their value within its depth range due to the rounding of the soundings. As a result, the depth contours are shifted towards the deeper side to traverse soundings with depths corresponding to the depth value of the depth contours displayed on the map.

All methods described have the following qualities: a. Criteria and constraints: The generalization procedure is based on IHO standards and NOAA best practices, b. Ladder approach: applying the ladder generalization approach to map compilation ensures intra layer (horizontal) and intra scale (vertical) consistency across scales, c. Flexibility and adaptability: the values of the parameters used, e.g., the spacing between soundings, fishnet size, the depth selection criteria, distance for aggregation, etc., can be set by the cartographer, resulting to a fully parameterized solution. It is considered that a "parametric" approach contributes significantly to the flexibility of the method and satisfies the requirements of different hydrographic institutions, d. Simplicity of implementation: The methodology is based on basic geographic data manipulation operations and the simple Open Geospatial Consortium (OGC) feature model. No special tools or special structures are required. Therefore, it can be implemented in any GIS environment and spatial database that provides these tools. This way, "in-house" GIS, existing databases and well-tested processing routines do not become obsolete. Consequently, the method can be easily adopted by HOs or private mapping companies, regardless of existing chart production procedures, e. Automation: As the generalization is automated to a considerable extent, the cartographer can only focus on checking the result of the automated procedure to deal with a small percentage of cases that may be omitted. This way, the speed of nautical chart production is increased and production costs are significantly reduced.

The suitability of the methods proposed has been verified by applying them to real data sets used by NOAA in nautical chart production. A case study (Skopeliti et al., 2020; Skopeliti et al., 2021) was conducted in the New York Lower Bay area based on a high-resolution DEM. The method successfully produced generalized shoreline, soundings and depth contours for a range of Harbour and Approach nautical charts at 1:10K, 1:20K, 1:40K, and 1:80K scales. Overall, it is noted that the proposed generalization method for soundings, shoreline and depth contours provides a holistic approach to the problem.

\section{Acknowledgements}

This research was funded by the University of New Hampshire, award number NA15NOS4000200-Subaward No: 19020 .

\section{References}

Guilbert, E. Multi-level representation of terrain features on a contour map. GeoInformatica 2012, 17, 301-324.

Skopeliti, A.; Stamou, L.; Tsoulos, L.; Pe'eri, S. Generalization of Soundings across Scales: From DTM to Harbour and Approach Nautical Charts. ISPRS Int. J. Geo-Inf. 2020, 9, 693. https://doi.org/10.3390/ijgi9110693

Skopeliti, A.; Tsoulos, L.; Pe'eri, S. Depth Contours and Coastline Generalization for Harbour and Approach Nautical Charts. ISPRS Int. J. Geo-Inf. 2021, 10, 197. https://doi.org/10.3390/ijgi10040197 